\title{
RESEARCH
}

Open Access

\section{Wound closure in epidermolysis bullosa: data from the vehicle arm of the phase 3 ESSENCE Study}

Dedee F. Murrell ${ }^{1}$, Amy S. Paller ${ }^{2 *}$, Christine Bodemer ${ }^{3}$, John Browning ${ }^{4}$, Milos Nikolic ${ }^{5}$, Jay A. Barth ${ }^{6}$, Hjalmar Lagast ${ }^{6}$, Eva Krusinska ${ }^{6+}$, and Allen Reha ${ }^{6}$ on behalf of the ESSENCE Study Group

\begin{abstract}
Background: Chronic wounds are a fundamental issue for patients with epidermolysis bullosa (EB). Herein, we assess the natural history of wound closure in patients with EB who were randomly assigned to the vehicle-control arm of the multicenter, randomized, double-blind, phase 3 ESSENCE (NCT02384460) trial.

Methods: ESSENCE was designed to assess the efficacy and safety of a topical cream formulation of $6 \%$ allantoin (SD-101 6\%) vs vehicle (SD-101 0\%) in patients $\geq 1$ month old who had a diagnosis of EB (simplex, recessive dystrophic, or intermediate junctional) and a target wound $10-50 \mathrm{~cm}^{2}$ present for $\geq 21$ days. Time to complete target wound closure and the proportion of patients with target wound closure over time were analyzed overall and by parameters including patient age and baseline body surface area index (BSAi) of total wound burden $(<5 \%$ and $\geq 5 \%$ ). Changes in BSAi of lesional skin, pain, and itching were also assessed.
\end{abstract}

Results: The vehicle-control arm included 87 patients. Mean (standard deviation [SD]) time to target wound closure within 3 months was 53.6 (28.6) days, with a range of 14 to 142 days. The proportion of patients with target wound closure increased over time from $7.1 \%$ at day 14 to $53.6 \%$ at month 3. Mean (SD) changes from baseline in BSAi of total wound burden and BSAi of lesional skin at month 3 were $-2.3 \%$ (6.3) and $-5.0 \%$ (13.5) of total body coverage, respectively. Reductions in pain and itching were observed at day 7 and maintained for 3 months. Faster healing times and a greater proportion of patients with wound closure were observed in patients aged 1 month to $<2$ years; those with wounds $<30$ days old, and in those with BSAi of total body wound burden $<5 \%$.

Conclusions: Treatment response observed in the vehicle-control arm of the ESSENCE study was unexpectedly high and may have been due to unforeseen benefits of vehicle or enhanced wound care provided by the clinical trial staff. These observations will help inform the study design of future trials in patients with EB.

Trial registration: ClinicalTrials.gov, NCT02384460; Date of registration: February 13, 2015; First participant enrollment: March 11, 2015.

Keywords: Epidermolysis bullosa, Wound closure, Natural history

\footnotetext{
* Correspondence: apaller@northwestern.edu

${ }^{\dagger}$ Author working as a consultant under the contract of Q5 Pharmaland

Consulting Group.

${ }^{2}$ Departments of Dermatology and Pediatric Dermatology, Northwestern

University Feinberg School of Medicine, 676 N. St. Clair, Suite 1600, Chicago,

IL 60611-2997, USA

Full list of author information is available at the end of the article
}

(c) The Author(s). 2020 Open Access This article is licensed under a Creative Commons Attribution 4.0 International License, which permits use, sharing, adaptation, distribution and reproduction in any medium or format, as long as you give appropriate credit to the original author(s) and the source, provide a link to the Creative Commons licence, and indicate if changes were made. The images or other third party material in this article are included in the article's Creative Commons licence, unless indicated otherwise in a credit line to the material. If material is not included in the article's Creative Commons licence and your intended use is not permitted by statutory regulation or exceeds the permitted use, you will need to obtain permission directly from the copyright holder. To view a copy of this licence, visit http://creativecommons.org/licenses/by/4.0/. The Creative Commons Public Domain Dedication waiver (http://creativecommons.org/publicdomain/zero/1.0/) applies to the data made available in this article, unless otherwise stated in a credit line to the data. 


\section{Introduction}

Epidermolysis bullosa (EB) is a genetically heterogeneous group of rare, devastating disorders characterized by fragility of skin and mucous membranes that blister in response to minor mechanical trauma [1-3]. Signs and symptoms of EB, which include chronic blisters or erosions, ulcers, severe itching, pain, and recurrent wound infection, typically appear in infancy and continue throughout life [4]. Blisters and erosions can occur anywhere on the body, but are typically found in areas of normal mechanical trauma, such as palms, soles, limbs, face, and diaper area [4]. Blisters can also occur in mucous membranes, including the mouth, which can limit food intake and cause scarring with stricture formation [4].

There are 4 main EB types based on the ultrastructural location of skin cleavage: the basal layer of the epidermis for the simplex type, the dermis for the dystrophic type, the epidermal-dermal junction for the junctional type, and multiple cleavage planes for Kindler syndrome [2, 4]. EB simplex is typically the mildest form of the disease and is generally associated with less scarring and fewer internal complications compared with junctional or dystrophic EB [2]. Severe junctional EB (generalized severe sub-type, formerly termed Herlitz sub-type) is associated with the highest risk of infant mortality, usually resulting from sepsis, failure to thrive, or tracheolaryngeal obstruction [2], whereas recessive dystrophic EB is associated with severe scarring and contractures that can substantially decrease physical function [2, 4, 5].

Currently there are no approved specific therapies for $E B$, and current standard of care involves cleaning and bandaging wounds and pain management [1, $2,6]$. Recommended treatments for wounds can vary depending upon disease severity, ranging from use of nonadherent wound dressings to topical creams and ointments, topical antiseptics, and topical and systemic antibiotics [1]. Wound care standards may also vary between countries or treatment centers, although consensus recommendations, published in 2014, aimed to harmonize and optimize the global management of EB [1].

The phase 3 ESSENCE trial (ClinicalTrials.gov: NCT02384460) was a 3-month, double-blind vehiclecontrolled trial designed to assess the efficacy and safety of SD-101, a cream formulation of $6 \%$ allantoin (SD-101 6\%), in patients with EB [7]. The primary, prespecified results of this study are reported in the companion paper to this article [8]. The inclusion of a vehicle arm (SD-101 with $0 \%$ allantoin) in this trial provides an important opportunity to gather data on the natural history of wound closure and other clinical endpoints in patients with EB. This analysis presents descriptive data on baseline wound characteristics and changes from baseline over 3 months of vehicle treatment in the ESSENCE trial, including analyses by patient age, EB type, and baseline wound characteristics (wound age and total body wound burden).

\section{Methods}

\section{Study design and participants}

Detailed methodology of the ESSENCE study is reported in the companion paper to this article [8]. In brief, the ESSENCE study was a multicenter, randomized, doubleblind, vehicle-controlled, phase 3 trial designed to assess the efficacy and safety of SD-101 6\% vs vehicle (SD-101 $0 \%)$ in patients with simplex, recessive dystrophic, or intermediate junctional EB. Eligible patients were $\geq 1$ month-old with a diagnosis of simplex, recessive dystrophic, or intermediate junctional EB and a target wound between 10 and $50 \mathrm{~cm}^{2}$ in size that had been present for $\geq 21$ days (based on patient history). Screening and baseline visits could be combined if patients were eligible. Patients were excluded if they had clinical evidence of local infection in the target wound, had used any investigational drug or systemic or topical steroidal therapy within the 30 days before enrollment (inhaled steroids and ophthalmic drops containing steroids were allowed), had used immunotherapy or cytotoxic chemotherapy within 60 days before enrollment, had used systemic antibiotics within 7 days before enrollment, had current or past malignancy, or had an arterial or venous disorder resulting in ulcerated lesions.

\section{Treatment}

Patients were randomly assigned 1:1 to receive SD$1016 \%$ or vehicle, which was applied topically in a thin layer over the entire body (including all nonwounded areas) once-daily along with daily bandage changes for 90 days using an interactive web response system. SD-101 6\% is a topical cream containing 6\% allantoin in an oil-in-water emulsion. The vehicle was the same cream formulation as SD-101 but excluding allantoin. SD101 and the vehicle contained the following excipients: beeswax, butylated hydroxytoluene, cetyl alcohol, citric acid, cod liver oil, lanolin oil, methylparaben, propylene glycol, propylparaben, sodium lauryl sulfate, stearyl alcohol, tetrasodium ethylenediaminetetraacetic acid, and purified water.

\section{Endpoints and assessments}

Primary endpoints were time to complete target wound closure (defined as skin re-epithelialization without drainage) within 3 months and the proportion of patients with target wound closure within 3 months. Key secondary endpoints were proportion of patients with target 
wound closure (at Months 1 and 2), evaluated using the ARANZ SilhouetteStar ${ }^{\text {TM }}$ device; change in body surface area index (BSAi) of lesional skin (percentage of total body coverage of EB-related lesions) and total body wound burden (percentage of total body coverage of open wounds) at Month 3; change from baseline in patient-reported pain using the Face, Legs, Activity, Cry, Consolability (FLACC) scale for patients aged 1 month to 3 years and the Wong-Baker FACES ${ }^{\circledR}$ Pain Scale for patients aged $\geq 4$ years $[9,10]$ at Day 7 ; change from baseline in patient-reported itching (via the Itch Man Pruritus Assessment Tool [11]) at Day 7; and the number and incidence of adverse events.

\section{Statistics}

The current analysis includes only data from the vehicle arm of the study, and descriptive results are presented. Demographics, baseline wound characteristics, time to complete wound closure, and the proportion of patients with target wound closure over time were analyzed overall and by patient age ( 1 month to $<2$ years, 2 to $<12$ years, 12 to $<18$ years, $\geq 18$ years); target wound age ( $\leq 30$ days and $>30$ days); baseline BSAi of total wound burden $(<5 \%$ and $\geq 5 \%)$; and EB type/subtype (simplex, intermediate junctional, and recessive dystrophic). Changes in BSAi of lesional skin, BSAi of total body wound burden, pain, and itching were evaluated over time in the overall vehicle-treated cohort.

\section{Results}

\section{Patients}

Of 169 patients enrolled in the trial, 87 were randomly assigned to the vehicle control group. Eighty patients (92\%) in the vehicle control group completed the study; reasons for discontinuation included adverse events $(n=2 ; 2.3 \%)$, withdrawal by patient $(n=3 ; 3.4 \%)$, and other $(n=2 ; 2.3 \%$; elective bone marrow transplantation $[n=1]$; could not attend study visits or comply with treatment application $[n=1])$.

The patient population was heterogeneous and represented a broad range of disease and demographic characteristics (Table 1; individual patient characteristics are presented in Additional file 1). At baseline, target wounds varied considerably in size. The mean (standard deviation [SD]) target wound size in the overall vehicle control group was $22.0(31.7) \mathrm{cm}^{2}$ and ranged from 7.8 to $302.0 \mathrm{~cm}^{2}$. Variations in target wound age were also observed, with patients aged $\geq 18$ years and those with intermediate junctional EB having the most chronic wounds (mean [SD] of 1115 [3350] days and 1676 [3822] days, respectively) (Table 2). Mean (SD) BSAi of total body wound burden was $10.5 \%$ (9.1), and most (55 of 87) patients at baseline had a BSAi of total body wound burden $\geq 5 \%$ (Table 2).

\section{Efficacy results in the overall vehicle control group}

In the overall vehicle control group, mean (SD) time to target wound closure within 3 months was 53.6 (28.6) days, with a median of 57.0 days and a range of 14 to 142 days. The proportion of patients with target wound closure increased over time from $7.1 \%$ at day 14 to $53.6 \%$ at month 3. Vehicle-treated patients experienced reductions in lesional skin and total body wound burden over the 3-month treatment period. Mean (SD) change from baseline in BSAi of lesional skin at month 3 was $-5.0 \%$ (13.5) of total body coverage (Fig. 1a). Mean (SD) change from baseline in BSAi of total wound burden at month 3 was $-2.3 \%$ (6.3) of total body coverage (Fig. 1b). Patients in the vehicle control group also experienced reductions from baseline in pain and itching at the first study visit (Day 7), which were maintained over the 3-month study period (Fig. 2a and b, respectively).

\section{Subgroup analyses for target wound closure Patient age}

Mean (SD) time to target wound closure was numerically shorter in patients aged 1 month to $<2$ years (43.8 [23.6] days) compared with patients in older age groups ( 2 to $<12$ years, 56.4 [27.8] days; 12 to $<18$ years, 53.4 [29.1] days; $\geq 18$ years, 52.8 [33.1] days). Differences in the proportions of patients with complete target wound closure over 3 months were also observed by age group, with the youngest age group ( 1 month to $<2$ years) having the largest proportion of patients attaining complete target wound closure at month 3 (83.3\%) (Fig. 3a).

\section{Target wound age}

Mean (SD) time to target wound closure was shorter for target wounds $\leq 30$ days old compared with those $>30$ days old (46.7 [22.2] days vs 60.7 [33.0] days). Twentythree of 24 (95.8\%) evaluable patients with target wounds $\leq 30$ days old achieved target wound closure within 3 months compared with 22 of 60 (36.7\%) evaluable patients with target wounds $>30$ days old (Fig. 3b).

\section{BSAi of total wound burden}

Mean (SD) time to complete wound closure was shorter in patients with BSAi of total wound burden $<5 \%$ compared with those with BSAi of total wound burden $\geq 5 \%$ (46.6 [25.4] days vs 60.9 [30.4] days). A higher proportion of patients with BSAi of total wound burden $<5 \%$ achieved target wound closure within 3 months compared with those who had BSAi of total wound burden $\geq 5 \%$ (73.3\% vs $41.5 \%$ ) (Fig. 3c).

\section{EB type}

Mean (SD) time to complete wound closure was shorter in patients with recessive dystrophic EB (49.4 [26.2] days) than in those with simplex EB (73.6 [16.6] days) or 


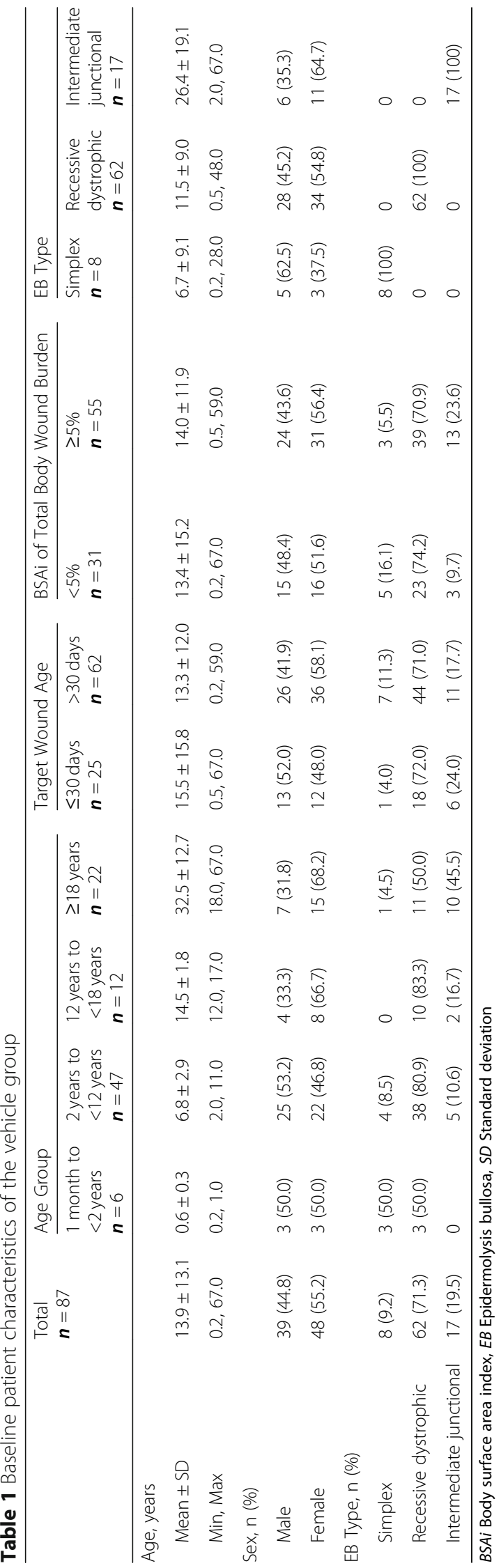




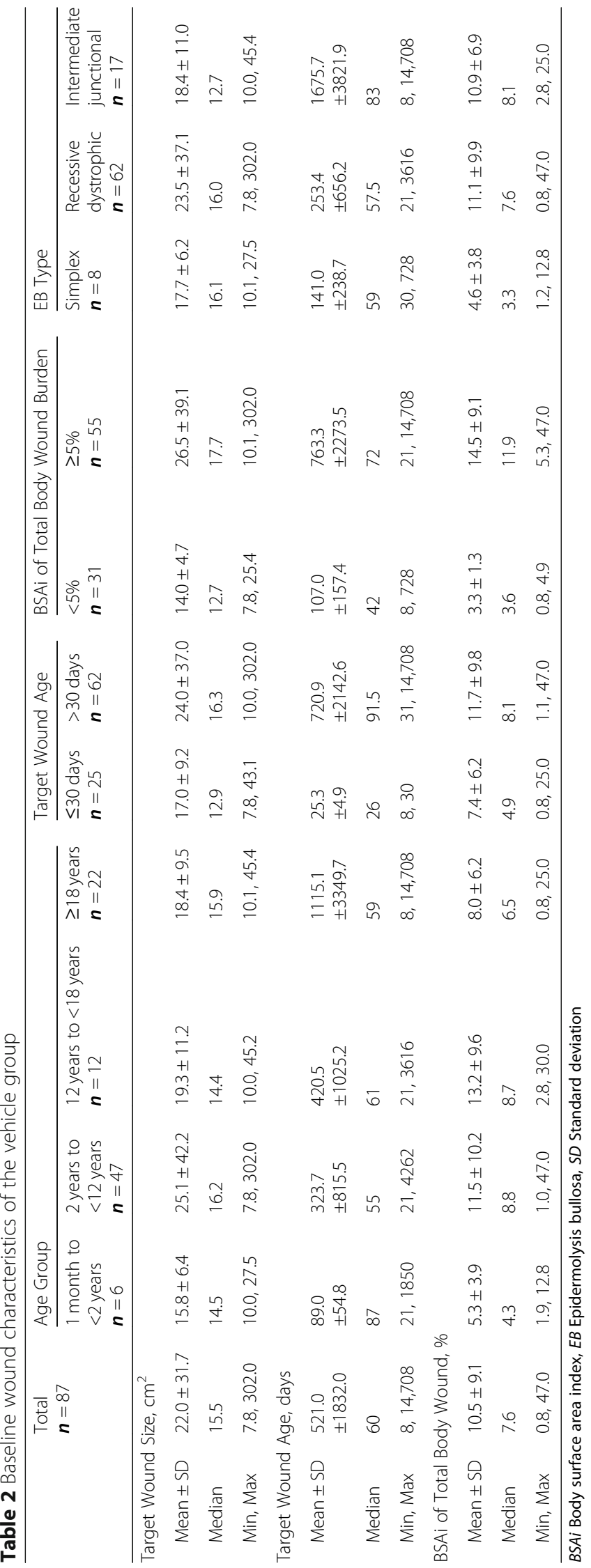




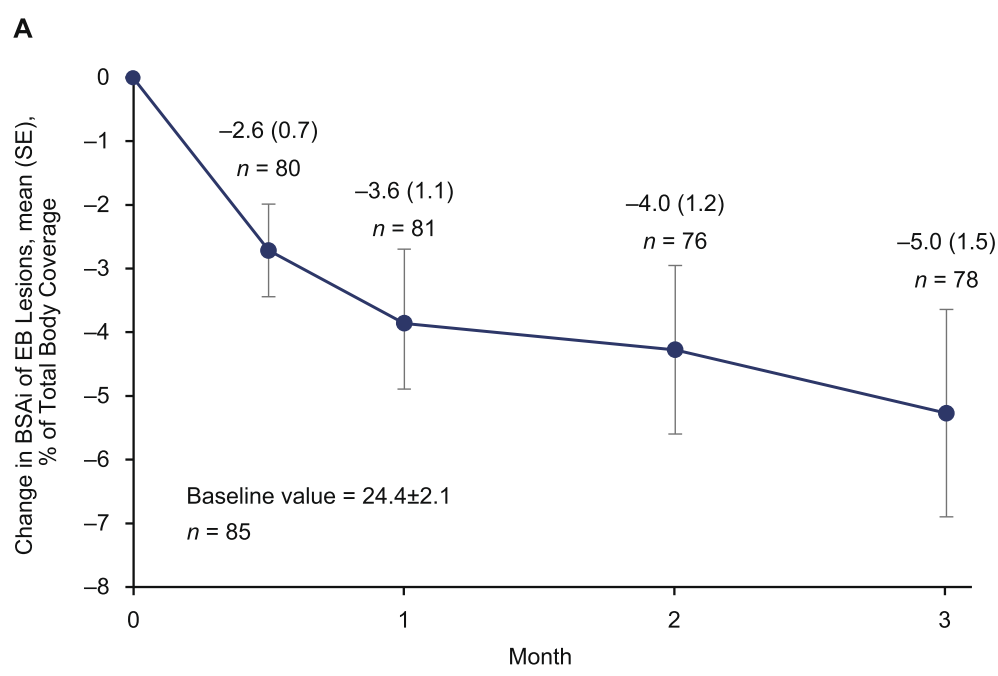

B

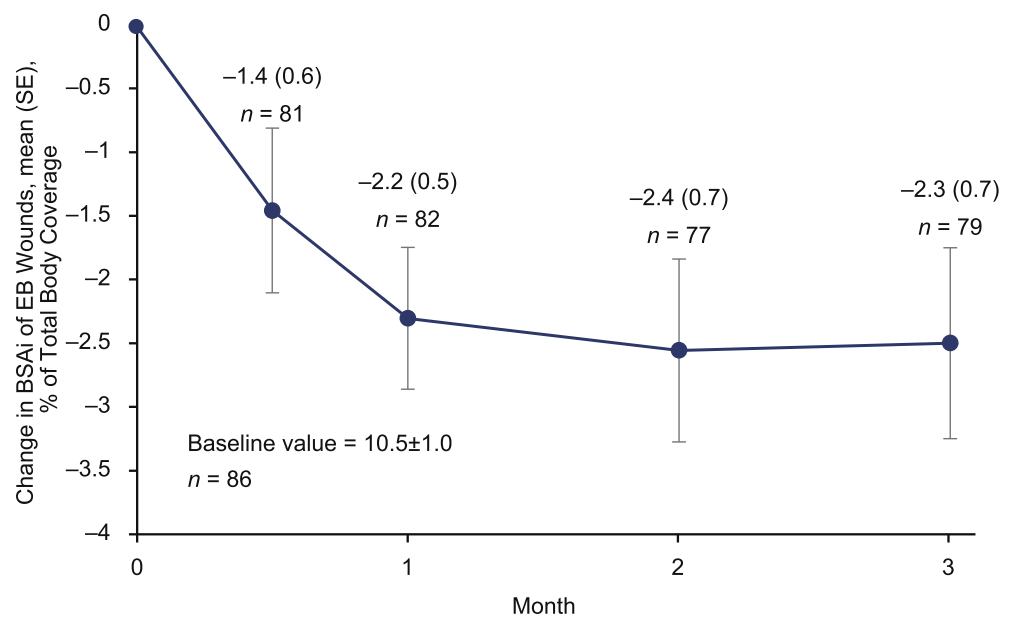

Fig. 1 Change in (a) lesional skin BSAi, (b) total body wound burden BSAi over 3 months. BSAi body surface area index; EB epidermolysis bullosa; SE standard error. Analysis excluded patients with missing values, resulting in variability in patient numbers at each visit

intermediate junctional EB (56.0 [37.0] days), although the proportion of patients with complete target wound closure was similar at month 3 for simplex and intermediate junctional and slightly lower for recessive dystrophic EB (simplex, 62.5\%; intermediate junctional, 58.8\%; recessive dystrophic, 50.8\%) (Fig. 3d).

\section{Discussion}

To our knowledge, this is the first study to report the natural history of wound closure in patients with $\mathrm{EB}$ who applied a vehicle (SD-101 0\%) over their entire body for 3 month's duration. The analysis evaluated the most common EB types, as well as patient age, baseline wound age, and baseline total body wound burden. The only other published report of a clinical trial assessing a topical cream in patients with EB in the past 20 years evaluated only the reduction of number of blisters by $>40 \%$ from baseline in selected areas and in just 17 patients with EB simplex [12]. The present study included 87 patients for the natural history arm, a remarkable sample size for a rare disease.

In the current study, the proportion of patients with each EB type in the vehicle-treated population was similar to those reported in epidemiology studies [13], and most vehicle-treated patients had a baseline BSAi of total wound burden of $\geq 5 \%$ at baseline. In general, wound healing time varied greatly (14 to 142 days), with the youngest patients ( 1 month to 2 years) having the fastest healing times and the greatest proportion of patients with complete wound closure by 3 months compared with other age groups. When including this young age group in future trials of wound healing in EB or/and other skin diseases, one must consider that rapid healing times may confound the ability to discriminate between 


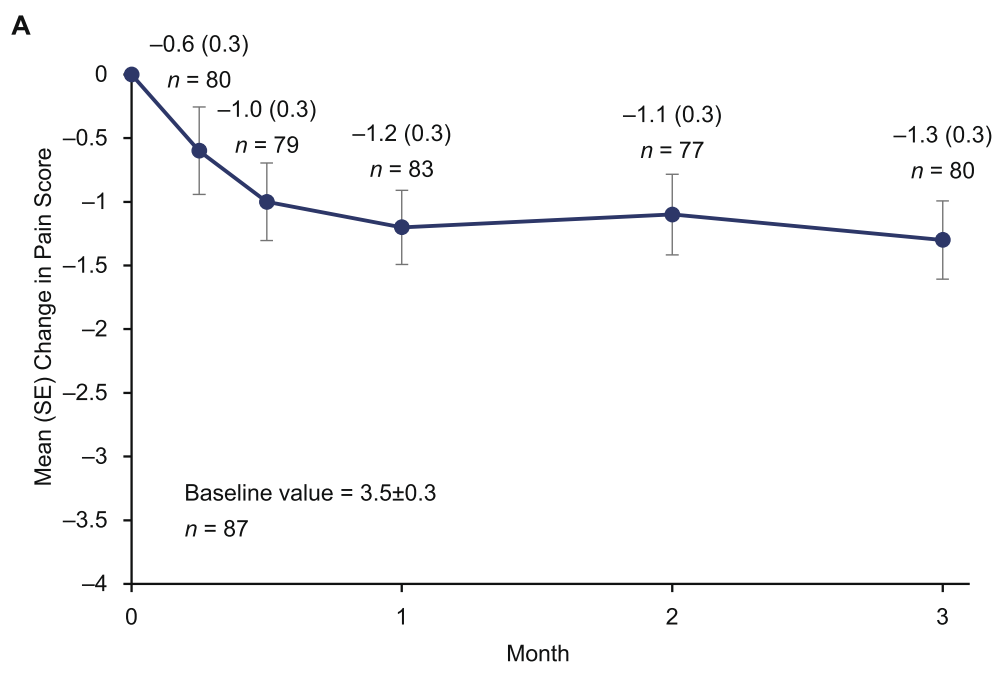

B

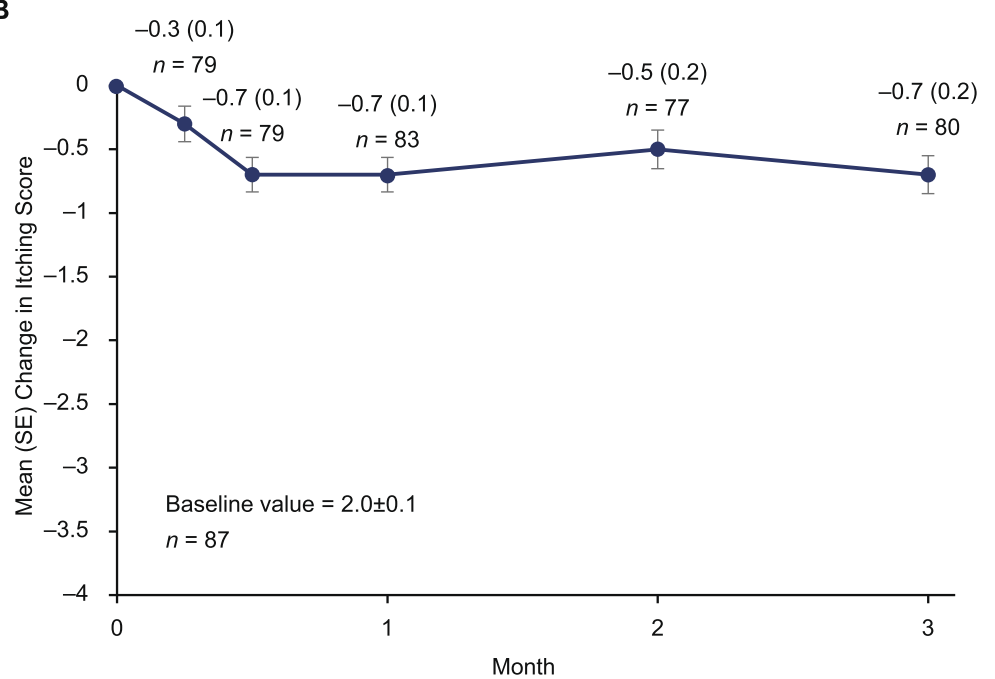

Fig. 2 Change in (a) pain and (b) itch scores over 3 months. SE standard error. Analyses excluded patients with missing data

active and control treatment arms. A positive aspect of treating young patients is that early healing may lead to fewer complications in this age group. However, due to the small sample size of the youngest age subgroup $(n=6)$, additional natural history data are needed to further understand the extent of differences between this age group versus older patients. In addition, wound closure was observed in a higher proportion of patients and at earlier timepoints in patients with relatively newer wounds ( $<30$ days old $)$ and in those with smaller total wound burden (BSAi $<5 \%$ ). These findings support the importance of patient stratification based on these factors.

It is notable that the proportion of patients with target wound closure within 3 months in vehicle-treated patients did not appear to differ greatly between EB types. These findings are surprising because differences in disease severity have been reported across EB types, with recessive dystrophic EB typically associated with greater disease severity and poorer outcomes compared with patients with EB simplex [2, 4]. It is worth noting that the EB simplex type can be further categorized into subtypes with varying severity [14]. EB simplex subtypes were not reported during the study and potential heterogeneity within EB simplex and the relatively fewer number of patients with EB simplex may have masked the differences between EB simplex and recessive dystrophic EB. Another surprising finding from the current study is that the efficacy response rate in the vehicle arm was much higher than anticipated for natural history, with approximately half of vehicle-treated patients achieving wound closure at 3 months. Natural history studies have shown that patients with recessive dystrophic EB suffer from chronic open wounds that typically last for years [15]. 


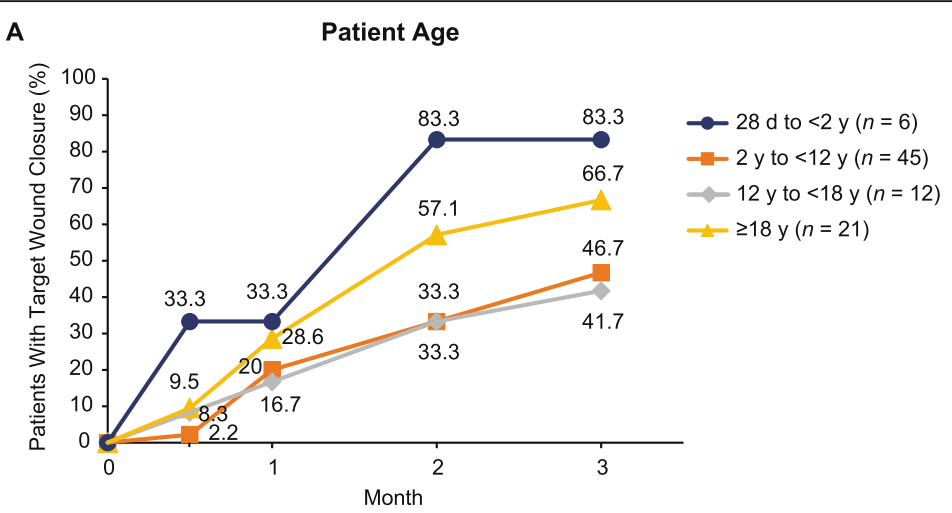

B

Target Wound Age

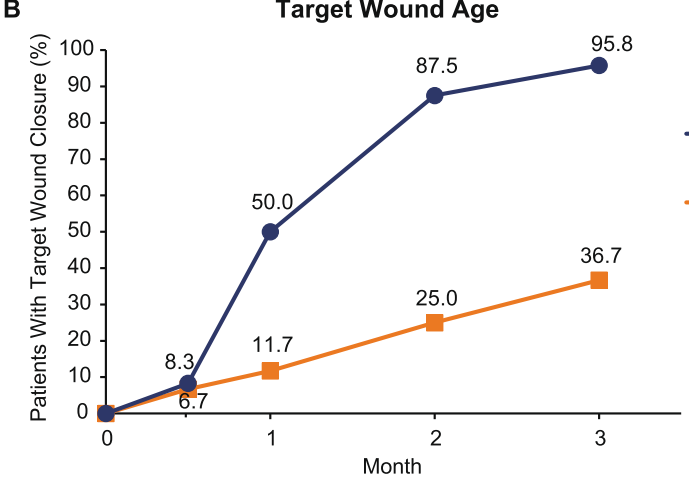

- Target Wound $\leq 30$ days $(n=24)$

- Target Wound

$>30$ days $(n=60)$

C

BSAi of Total Body Wound Burden

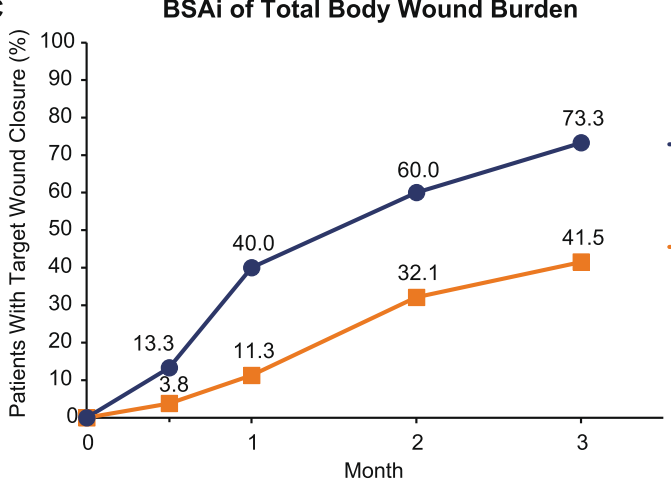

- BSAi of Total Body

Wound Burden

$<5 \%(n=30)$

- BSAi of Total Body

Wound Burden

$\geq 5 \%(n=53)$

D

EB Type

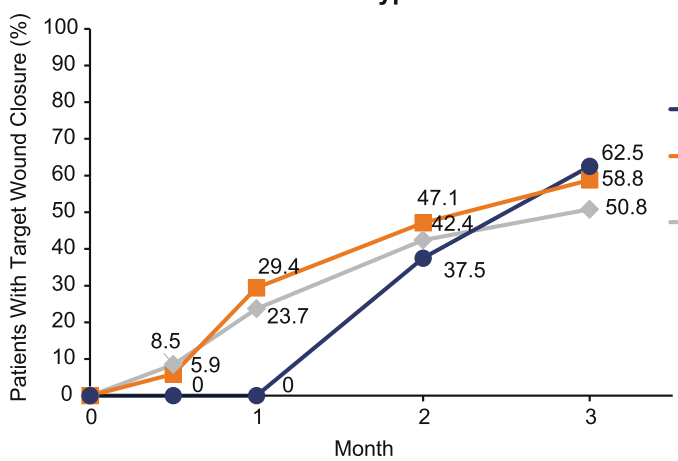

EB Simplex $(n=8)$

- Intermediate junctional EB $(n=17)$

- Recessive dystrophic $\mathrm{EB}(n=59)$

Fig. 3 Proportion of vehicle-treated patients with complete target wound closure within 3 months by (a) patient age, (b) target wound age, (c) BSAi of total body wound burden, and (d) EB type. BSAi body surface area index; $d$ day; EB epidermolysis bullosa; $y$ year 
For comparison, the mean time to wound closure in vehicle-treated patients with recessive dystrophic EB in the present study was approximately 7 weeks, with approximately $50 \%$ of patients with dystrophic EB achieving wound closure at 3 months.

Several limitations of our study should be considered when interpreting the data. First, there was no statistical comparison between subgroups. Second, patients in this analysis were drawn from a clinical trial that was not designed to evaluate natural history. Third, the number of patients in this study was not balanced across EB types because patients with recessive dystrophic EB most often met the criteria for inclusion in the study. Fourth, daily application of an oil-in-water emulsion cream to the entire body, including areas of nonwounded skin, does not reflect current skin care recommendations for patients with EB [1]. The oil-in-water formulation of the vehicle cream contained excipients including lanolin oil and cod liver oil that may have contributed to the efficacy response observed [16, 17]. Furthermore, daily dressing changes and careful physician monitoring may have contributed to the efficacy response. In the present study, patient visits were typically $3 \mathrm{~h}$ in length, and the attention to wound care received by the patients may have been greater than the routine normally practiced at home. In a small, randomized, vehicle-controlled trial of $1 \%$ diacerein topical cream in patients with EB simplex conducted by Wally and colleagues, the authors attributed a small placebo effect in the control arm to regular and intensive wound care received during the study period [12]. Because at-home wound care routines and the frequency of physician visits before study initiation were not collected in sufficient detail, we cannot determine how trial-related activities may have influenced efficacy assessments in the vehicle control group. In addition, patient expectations and beliefs about a potential treatment and the possibility of a beneficial outcome can also cause a "placebo effect," an observation that is becoming increasingly apparent in dermatology [18]. Given the significant burden of EB, and the lack of an approved therapy, it is reasonable to assume that both patient and caregiver's expectations of treatment in the present study was high. Finally, there was no control group that included patients who practiced standard of care or their "usual" wound care routine that could be compared to the vehicle-control group.

\section{Conclusions}

Treatment response observed in the vehicle-control arm of the ESSENCE study was unexpectedly high, with overall improvements observed in target wound closure, lesional skin and total body wound burden, itch, and pain over the 3-month treatment period. The reason for these improvements could be due to several factors, including unforeseen beneficial effects of the oil-in-water formulation or other excipients in the topical cream, as well as enhanced wound care provided by clinical trial staff. Patients aged $<2$ years, patients with target wounds $\leq 30$ days old, and patients with BSAi of total wound burden $<5 \%$ tended to have shorter time to target wound closure and greater wound closure rate within 3 months while receiving vehicle treatment. These observations are of considerable interest and may help inform the study design of future trials in patients with $\mathrm{EB}$, which may benefit from a vehicle run-in phase to exclude patients who respond to vehicle controls or improved wound care.

At present, wound care in patients with EB should conform to current treatment guidelines, with consistency across treatment centers, to optimize management of this condition. However, topical application of an emollient cream to both wounded and nonwounded skin, especially with frequent dressing changes, may encourage barrier repair in patients with EB. Such a management strategy may reduce itch and further trauma to existing wounds, preserve nonwounded skin and, hence, prove useful in the management of patients with EB.

\section{Supplementary information}

Supplementary information accompanies this paper at https://doi.org/10. 1186/s13023-020-01435-3.

Additional file 1. ESSENCE individual baseline characteristics of the vehicle group. Contains individual de-identified data of patients who were randomly assigned to the vehicle group in the current study.

\section{Abbreviations}

BSAi: Body surface area index; EB: Epidermolysis bullosa; FLACC: Face, Legs, Activity, Cry, Consolability scale; SD: Standard deviation; SE: Standard error

\section{Acknowledgments}

The authors thank the patients and their families, as well as the study investigators. Third-party medical writing assistance was provided by SallyAnne Mitchell, PhD, Lynn Brown, PhD, and Lei Bai, PhD, of ApotheCom (Yardley, PA) and was supported by Amicus Therapeutics, Inc.

\section{Authors' contributions}

$A S P, A R, H L$, and JAB participated in study design; DFM, ASP, CB, JB, MN, and $\mathrm{HL}$ assisted in the acquisition of data; $\mathrm{ASP}, \mathrm{HL}$, and EK analyzed data; ASP, $H L$, and JAB interpreted the data; HL drafted the manuscript. All authors critically revised the manuscript; all authors gave final approval of the submitted version and agreed to be accountable for all aspects of the work in ensuring that questions related to the accuracy or integrity of any part of the work are appropriately investigated and resolved.

\section{Funding}

The study was supported by Amicus Therapeutics, Inc. This study was designed, managed, and analyzed jointly by authors employed by Amicus Therapeutics, Inc. and external authors who were not paid for their work.

\section{Availability of data and materials}

The data supporting the findings of this study are reported in the companion manuscript (entitled "Efficacy and tolerability of the investigational topical cream SD-101 [6\% allantoin] in patients with epidermolysis bullosa: a phase 3 , randomized, double-blind, vehicle-controlled trial 
[ESSENCE Study])" and its additional file, which was submitted contemporaneously to Orphanet Journal of Rare Diseases.

\section{Ethics approval and consent to participate}

The study was conducted in accordance with ethical principles founded in the Declaration of Helsinki and in compliance with the protocol, International Conference on Harmonisation Good Clinical Practice, and applicable local legal and regulatory requirements. An Institutional Review Board/Independent Ethics Committee reviewed and approved the study design and protocol at each study site and signed informed consent/assent was obtained from each patient (or legal guardian if under 18 years of age) prior to entering the study.

\section{Consent for publication}

Not applicable.

\section{Competing interests}

DFM has served as a consultant and investigator for Amicus, Amryt, Castle Creek Pharma, and Shire and has conducted her own investigator-initiated randomized controlled trials in recessive dystrophic epidermolysis bullosa and epidermolysis bullosa simplex. ASP has served as an investigator (uncompensated) for Amicus, Castle Creek Pharmaceuticals, Lenus Therapeutics and Shire, and as a consultant with honorarium for Castle Creek Pharmaceuticals, Lenus Therapeutics, and MEDACorp. CB has served on advisory boards for Pfizer and Sanofi, as a consultant for Expanscience, as an investigator (uncompensated) for Amicus, as a speaker for La Roche-Posay and Expanscience, and has received honoraria from Celgene and Novartis. JB has served as an investigator for Amicus, Galderma, Valeant, Patagonia, and Regeneron and as a speaker for Medimetriks and Promius. MN has received honoraria from Meda/Mylan, Medis, La Roche-Posay/L'Oreal, Uriage, Takeda, Novartis, Janssen, and Amicus. JAB, HL, and AR are employees of and hold stock in Amicus. EK is a paid consultant to Amicus.

\section{Author details}

'University of New South Wales, Sydney, NSW, Australia. ²Departments of Dermatology and Pediatric Dermatology, Northwestern University Feinberg School of Medicine, 676 N. St. Clair, Suite 1600, Chicago, IL 60611-2997, USA. ${ }^{3}$ EB Expert Centre (MAGEC), Department of Dermatology, Necker-Enfants Malades Hospital, Paris Centre University, Paris, France. ${ }^{4}$ Texas Dermatology \& Laser Specialists, San Antonio, TX, USA. ${ }^{5}$ Clinical Center of Serbia, Department of Dermatology, University of Belgrade, Belgrade, Serbia. ${ }^{6}$ Amicus

Therapeutics, Inc., Cranbury, NJ, USA.

Received: 20 February 2020 Accepted: 5 June 2020

Published online: 21 July 2020

\section{References}

1. El Hachem M, Zambruno G, Bourdon-Lanoy E, Ciasulli A, Buisson C, HadjRabia S, et al. Multicentre consensus recommendations for skin care in inherited epidermolysis bullosa. Orphanet J Rare Dis. 2014;9:76.

2. Fine JD. Inherited epidermolysis bullosa. Orphanet J Rare Dis. 2010;5:12.

3. Berk DR, Jazayeri L, Marinkovich MP, Sundram UN, Bruckner AL. Diagnosing epidermolysis bullosa type and subtype in infancy using immunofluorescence microscopy: the Stanford experience. Pediatr Dermatol. 2013;30(2):226-33.

4. Tabor A, Pergolizzi JV Jr, Marti G, Harmon J, Cohen B, Lequang JA. Raising awareness among healthcare providers about epidermolysis bullosa and advancing toward a cure. J Clin Aesthet Dermatol. 2017;10(5):36-48.

5. Eismann EA, Lucky AW, Cornwall R. Hand function and quality of life in children with epidermolysis bullosa. Pediatr Dermatol. 2014;31(2):176-82.

6. Goldschneider KR, Good J, Harrop E, Liossi C, Lynch-Jordan A, Martinez AE, et al. Pain care for patients with epidermolysis bullosa: best care practice guidelines. BMC Med. 2014;12:178.

7. ESSENCE Study: Efficacy and Safety of SD-101 Cream in Patients With Epidermolysis Bullosa. https://clinicaltrials.gov/ct2/show/NCT02384460. Last updated 15 November 2018. Accessed 13 December 2019.

8. Paller AS, Browning J, Nikolic M, Bodemer C, Murrell DF, Lenon W, et al. Efficacy and tolerability of the investigational topical cream SD-101 (6\% allantoin) in patients with epidermolysis bullosa: a phase 3, randomized, double-blind, vehicle-controlled trial (ESSENCE study). Orphanet J Rare Dis. 2020;15:158
9. Merkel SI, Voepel-Lewis T, Shayevitz JR, Malviya S. The FLACC: a behavioral scale for scoring postoperative pain in young children. Pediatr Nurs. 1997; 23(3):293-7.

10. Wong DL, Baker CM. Smiling faces as anchor for pain intensity scales. Pain. 2001;89(2-3):295-300.

11. Morris V, Murphy LM, Rosenberg M, Rosenberg L, Holzer CE 3rd, Meyer WJ 3rd. Itch assessment scale for the pediatric burn survivor. J Burn Care Res. 2012;33(3):419-24.

12. Wally V, Hovnanian A, Ly J, Buckova H, Brunner V, Lettner T, et al. Diacerein orphan drug development for epidermolysis bullosa simplex: A phase 2/3 randomized, placebo-controlled, double-blind clinical trial. J Am Acad Dermatol. 2018;78(5):892-901.e7.

13. DEBRA UK. What is EB. https://www.debra.org.uk/what-is-eb/what-is-eb. Accessed 2 June 2020

14. Has C, Bauer JW, Bodemer C, Bolling MC, Bruckner-Tuderman L, Diem A, et al. Consensus reclassification of inherited epidermolysis bullosa and other disorders with skin fragility. Br J Dermatol. 2020. https://doi.org/10.1111/bjd. 18921 Online ahead of print.

15. Solis D, Nazaroff J, Dutt-Singkh Y, Choi S, Barriga M, Li S, et al. 572 defining chronic wound types in recessive dystrophic epidermolysis bullosa patients for clinical outcome assessment. J Investig Dermatol. 2018;138(5):S97.

16. Chvapil M, Gaines JA, Gilman T. Lanolin and epidermal growth factor in healing of partial-thickness pig wounds. J Burn Care Rehabil. 1988;9(3):279-84.

17. Terkelsen LH, Eskild-Jensen A, Kjeldsen H, Barker JH, Hjortdal VE. Topical application of cod liver oil ointment accelerates wound healing: an experimental study in wounds in the ears of hairless mice. Scand J Plast Reconstr Surg Hand Surg. 2000;34(1):15-20.

18. Evers AW. Using the placebo effect: how expectations and learned immune function can optimize dermatological treatments. Exp Dermatol. 2017;26(1): $18-21$.

\section{Publisher's Note}

Springer Nature remains neutral with regard to jurisdictional claims in published maps and institutional affiliations.

Ready to submit your research? Choose BMC and benefit from:

- fast, convenient online submission

- thorough peer review by experienced researchers in your field

- rapid publication on acceptance

- support for research data, including large and complex data types

- gold Open Access which fosters wider collaboration and increased citations

- maximum visibility for your research: over $100 \mathrm{M}$ website views per year

At BMC, research is always in progress.

Learn more biomedcentral.com/submissions 